\title{
Utility of magnetic resonance imaging in determining treatment response and local recurrence in nasopharyngeal carcinoma treated curatively
}

\author{
Katherine Meng ${ }^{*}$ (D), Jeremy Tey, Francis Cho Hao Ho, Hira Asim and Timothy Cheo
}

\begin{abstract}
Background: To determine the optimal timing of the first Magnetic Resonance Imaging (MRI) scan after curativeintent radiotherapy (RT) for nasopharyngeal carcinoma (NPC), and evaluate the role of MRI in surveillance for locoregional recurrence (LRR).

Methods: Patients with non-metastatic NPC treated radically who had at least one post-treatment MRI (ptMRI) done were included for analysis. ptMRI reports were retrospectively reviewed and categorised as complete response (CR), partial response/residual disease (PR) or indeterminate (ID). Patients with LRR were assessed to determine if initial detection was by MRI or clinical means. Univariable and multivariable Cox proportional hazard regression analysis were performed to identify independent factors associated with CR on ptMRIs.

Results: Between 2013 and 2017, 262 eligible patients were analysed, all treated with Intensity Modulated Radiotherapy (IMRT). Median time from end of RT to the first ptMRI was 93 days (range 32-346). Of the first ptMRIs, 88 (33.2\%) were CR, 133 (50.2\%) ID, and 44 (16.6\%) PR. A second ptMRI was done for 104 (78.2\%) of 133 patients with ID status. In this group, 77 (57.9\%) of the subsequent MRI were determined to be CR, 21(15.8\%) remained ID and 6 (4.5\%) PR. T1 tumour stage and AJCC stage I were associated with increased CR rates on first ptMRI on multivariable analysis. ID status was more likely at 75-105 days ( 3 months $+/-15$ days) vs 106-135 days ( 4 months $+/-15$ days) post RT (OR 2.13, 95\% Cl 1.16-4.12, $p=0.024$ ). LRR developed in 27 (10.1\%) patients; 20 (74.1\%) were initially detected through MRI, 3 (11.1\%) by nasoendoscopy and 2 (7.4\%) by PET-CT.

Conclusion: MRI is useful for detecting local recurrence or persistent disease after curative-intent treatment. Most patients will need more than one ptMRI to arrive at a definitive status. The rate of ID ptMRI may be reduced by delaying the first scan to around 4 months post RT.
\end{abstract}

Keywords: Nasopharyngeal carcinoma, Nasopharyngeal neoplasms, Radiotherapy, Intensity-modulated radiotherapy, Magnetic resonance imaging

\footnotetext{
* Correspondence: tian.meng@mohh.com.sg

Department of Radiation Oncology, National University Cancer Institute, 5

Lower Kent Ridge Road, Singapore 119074, Singapore
}

(c) The Author(s). 2020 Open Access This article is licensed under a Creative Commons Attribution 4.0 International License, which permits use, sharing, adaptation, distribution and reproduction in any medium or format, as long as you give appropriate credit to the original author(s) and the source, provide a link to the Creative Commons licence, and indicate if changes were made. The images or other third party material in this article are included in the article's Creative Commons licence, unless indicated otherwise in a credit line to the material. If material is not included in the article's Creative Commons licence and your intended use is not permitted by statutory regulation or exceeds the permitted use, you will need to obtain permission directly from the copyright holder. To view a copy of this licence, visit http://creativecommons.org/licenses/by/4.0/ The Creative Commons Public Domain Dedication waiver (http://creativecommons.org/publicdomain/zero/1.0/) applies to the data made available in this article, unless otherwise stated in a credit line to the data. 


\section{Background}

Nasopharyngeal Carcinoma (NPC) has a distinct ethnic and geographical distribution, and is common in Southern Chinese and South East Asian populations [1]. Nonmetastatic NPC is treated definitively with radiotherapy (RT) with or without chemotherapy. Treatment response has been closely associated with prognosis [2, 3]. Magnetic Resonance Imaging (MRI)'s role in the initial staging of biopsy-proven NPC is well established [4-7]. However, the utility of MRI in the assessment of response to treatment and disease surveillance is less welldefined.

The time course of histological NPC regression has previously been demonstrated through serial biopsy to be around 12 weeks post treatment with 3-dimensional conformal RT (3D-CRT) [8]. As such, the first assessment of treatment response is typically scheduled at this time-point through a combination of clinical and endoscopic examination with cross-sectional imaging. In practice, owing to their invasive nature, biopsies are usually reserved for cases where there are suspicious endoscopic or radiological findings. This imparts greater importance for imaging modalities such as MRI to pick up persistent or recurrent disease in a timely and accurate fashion.

Through this retrospective study, we explore the reporting patterns of post treatment MRI (ptMRI) for NPC patients, and aim to determine the optimal timing for the first ptMRI in a real-life clinical setting. In addition, we review current evidence and evaluate the ability of MRI compared to other clinical or radiological surveillance modalities in detecting locoregional recurrences (LRR).

\section{Methods}

\section{Patients}

Approval for this study was obtained from the Institutional Review Board (IRB). The NPC database, comprising all patients with histologically-proven NPC treated in two tertiary hospitals in Singapore, was retrospectively reviewed. Patients with non-metastatic NPC of any histological subtype treated with curative intent by RT alone, concurrent chemoradiation (CCRT) with or without induction chemotherapy between February 2013 and July 2017 were included for analysis. Pre-treatment evaluation for all patients included a complete history and physical examination, endoscopic assessment with biopsy, and staging scans. Computed Tomography (CT) or MRI was used for local staging, positron-emission tomography (PET)-CT was done to exclude metastatic disease. The staging system used was the American Joint Committee on Cancer (AJCC) 7th edition. Histology was classified according to the World Health Organisation system. For inclusion, at least one post-treatment MRI
(ptMRI) needed to be done within 1 year of RT completion. Patients who did not complete the prescribed course of RT or have ptMRIs done and reported in other local/overseas institutions were excluded.

\section{Treatment}

According to institutional guidelines, patients with stage I and node-negative stage II disease were treated with RT only. Node-positive stage II, stage III to IVB patients were treated with CCRT; those with T4 or N3 disease were also offered 2-3 cycles of induction chemotherapy. All patients received Intensity Modulated Radiotherapy (IMRT). CT simulation was done with administration of intravenous contrast; fusion with pre-treatment MRI was done for planning wherever possible. Treatment was carried out with patients in a supine position immobilised by a thermoplastic shell. Our treatment protocol closely follows that used in the Radiation Therapy Oncology Group (RTOG) 0615 trial [9]. All patients were prescribed a total dose of 69.96Gy in 33 fractions. Clinical Target Volume (CTV) is designated as the Gross Tumour Volume (GTV) with a circumferential margin of $\geq 5 \mathrm{~mm}$; where tumour is in close proximity to critical structures such as the optic apparatus or brain stem this margin may be reduced accordingly. $\mathrm{CTV}_{70}$ included gross disease in the nasopharynx and any overtly involved lymph nodes. High risk and low risk subclinical regions as outlined in the RTOG 0615 radiotherapy schema were prescribed $59.4 \mathrm{~Gy}$ and $54 \mathrm{~Gy}$ respectively. Concurrent weekly cisplatin-based chemotherapy (30-40 $\mathrm{mg} / \mathrm{m}^{2}$ ) was administered during RT. Treatment breaks were not specifically recorded.

\section{MR imaging, interpretation and timing}

Timing of ptMRIs was calculated from the last day of RT. As per institutional practice, all scans were either reported or verified by a radiologist with a special interest in head and neck imaging. There were minor variations in the MRI protocol between the two tertiary hospitals but in all patients, four key sequences were performed: axial T1-weighted, axial T1-weighted contrast-enhanced fat-suppressed, axial T2-weighted and diffusion-weighted series.

The first ptMRI reports were reviewed and coded as 'complete response' (CR), 'partial response' (PR) or 'indeterminate' (ID). For the first ptMRI, CR is defined as complete resolution of disease or absence of any residual tumour in both primary and nodal sites in the radiology report. In subsequent ptMRIs, CR also included stable posttreatment changes seen on later imaging. PR is defined as the presence of residual disease in either the primary and/ or affected nodes. Where the report indicated inability to definitively distinguish between residual tumour and post- 
treatment changes, or suggested clinical/endoscopic correlation, or repeat imaging, it was coded as ID.

To determine the optimal timing of first ptMRI, we arbitrated a cut-off point of 4 months +/- 15 days (106135) compared with 3 months +/- 15 days (75-105). Based on current institutional practice, the first ptMRI is typically done at 3 months post RT. However, in our experience the rate of indeterminate outcomes is high when the scan is performed at this time point. We hypothesised that delaying the timing of the first ptMRI by 1 month (or 30 days), the proportion of ID ptMRIs may be reduced. This particular value was selected so as not to deviate too far from the common practice of 3month post-RT scans, whilst keeping in mind that further delays to first ptMRIs may compromise timely detection of residual disease.

\section{Follow-up}

Depending on extent of acute toxicity, patients were reviewed at weeks 2 and 4 post RT. Subsequently the follow-up schedule would be 2 to 3-monthly until end of year 2, 4-monthly in year 3 and 6-monthly in years 4 to 5. Post-treatment review comprises clinical and endoscopic examinations, and may be shared between the patient's Ear, Nose \& Throat (ENT) surgeon or medical oncologist where appropriate. Although there is a lack of specific guidelines with regards to the schedule of post treatment imaging, the first ptMRI was typically done at around the 3-month mark. Subsequent ptMRIs were not mandated if the first scan was reported as a CR; but were ordered where clinically indicated, for example in patients with PR/ID on first ptMRIs, or those with suspicious clinical or endoscopic findings. Patients with LRR based on ptMRIs were identified and reviewed to determine the timing of recurrence, how it was first detected (clinically versus radiologically), and how it was managed. The follow-up period was calculated from the last day of RT to day of last medical encounter or death.

\section{Statistical analysis}

Descriptive analysis was done using frequency with percentage and median. Univariable Cox proportional hazard regression analysis was performed to look for association between various patient, disease and treatment characteristics with achieving CR on first and subsequent ptMRIs. Covariables with $P$-value of $\leq 0.05$ in the univariable analysis were included in the multivariable Cox proportional hazard regression analysis to identify independent factors associated with CR. For all analyses, two-sided $P$-values of $<0.05$ were considered to be statistically significant. Statistical analyses were performed using STATA version 14.0.

\section{Results}

Two hundred sixty-two patients treated between February 2013 and July 2017 were eligible for analysis (Table 1). 196 (74.8\%) were males and the median age at diagnosis is 55 (range 15-82). The most common histological subtype was undifferentiated non-keratinising (WHO Type III), in 243 patients (92.8\%). 70 (26.8\%) patients had stage I or II disease. Under TNM classification, $83(31.7 \%)$ patients had T4 and $43(16.4 \%)$ had N3 disease respectively. 51 (19.5\%) patients received RT alone. The rest received chemotherapy in combination with RT: 124 (47.3\%) underwent CCRT, 87 (33.2\%) had induction chemotherapy followed by CCRT. All patients received IMRT to the full prescribed dose of 69.96Gy in 33 fractions.

All 262 patients had at least one ptMRI. Of which, 86 (32.8\%) were reported as CR, $133(50.8 \%)$ as ID and 43 (16.4\%) as PR. In the 133 patients whose first ptMRI was reported as indeterminate, 104 (78.2\%) went on to have a second ptMRI (the remaining 29 patients had no further scans). In this group, 77 (57.9\%) of the second scans were reported as CR, 21 (15.8\%) remained ID and 6 (4.5\%) were PR (Fig. 1).

Multivariable analysis showed that T1 stage and AJCC Stage I were significantly associated with achieving CR on the first ptMRI (Odds Ratio [OR] 2.96, $p=0.036$ and OR $1.78, p=0.046$ respectively). Receiving CCRT was approaching significance (OR 2.46, $p=0.054$ ) (Table 2).

The median time from end of RT to having the first ptMRI was 93 days (range 32-346). ID status was more likely if the scan was done between 75 and 105 days (3 months +/ -15 days) compared to 106-135 days (4 months +/- 15 days) post RT (OR 2.13, 95\% CI 1.164.12, $p=0.024$ ) (Table 3).

The median follow-up duration was 2.36 years (range 0.08-4.6). LRR was detected in 27 (10.1\%) patients during follow-up. Of these, 20 (74.1\%) were initially picked up by ptMRIs, $3(11.1 \%)$ were detected on nasoendoscopy and $2(7.4 \%)$ by PET-CT. In this group of patients, 16 (59.3\%) had local recurrence, 7 (25.9\%) had regional recurrence in neck nodes, 4 (14.8\%) had synchronous local and regional recurrences.

\section{Discussion}

Outcomes for patients with NPC have improved over the years with the introduction of chemotherapy and IMRT. However, local failure in the form of residual or recurrent disease still occur in $10-30 \%$ of cases $[10,11]$. The assessment of treatment response and clinical surveillance after definitive therapy for NPC is important, in order to permit earlier recognition of local failure and initiation of salvage therapies. Typically, patients are assessed clinically with cranial nerve examination, neck palpation and endoscopic inspection, in combination 
Table 1 Patient and tumour characteristics

\begin{tabular}{|c|c|c|c|c|c|}
\hline \multicolumn{2}{|l|}{ Patient characteristics } & \multicolumn{4}{|l|}{ Tumour characteristics } \\
\hline Number of patients & 262 & Histology & No. (\%) & T-stage & No. (\%) \\
\hline Age at diagnosis (range) & 55 (15-82) years & Non-keratinising undifferentiated & $243(92.7)$ & 1 & $90(34.4)$ \\
\hline Gender & No. (\%) & Non-keratinising differentiated & $10(3.8)$ & 2 & $25(9.5)$ \\
\hline Male & $196(74.8)$ & Keratinising & $2(0.8)$ & 3 & $64(24.4)$ \\
\hline Female & $66(25.2)$ & Others & $7(2.7)$ & 4 & $83(31.7)$ \\
\hline Ethnicity & No. (\%) & AJCC Stage ( $7^{\text {th }}$ edition) & No. (\%) & N-Stage & No. (\%) \\
\hline Chinese & $207(79.0)$ & । & $23(8.8)$ & 0 & $38(14.5)$ \\
\hline Malay & $31(11.8)$ & $\|$ & $47(18.0)$ & 1 & $90(34.4)$ \\
\hline Indian & $4(1.5)$ & III & $80(30.5)$ & 2 & $93(35.5)$ \\
\hline Others & $20(7.7)$ & IV A/B & $112(42.7)$ & 3 & $41(15.6)$ \\
\hline
\end{tabular}

with imaging such as CT, MRI or PET-CT. Any suspicious lesions are then biopsied to obtain histological confirmation.

Given that radiation can lead to anatomical distortion within the treatment field, identification of residual or recurrent disease is often challenging. Palpation for cervical lymph nodes may be limited by fibrosis of neck musculature. Post radiation endoscopic examination may only reveal subtle mucosal changes such as fullness of postnasal space (PNS), or a mass which may represent fibrosis, crust or slough rather than residual tumour
[12], and submucosal or deep-seated recurrences may be missed. Sensitivity of endoscopic examination in detecting persistent disease after RT is only $40.4 \%$. Similarly, endoscopic biopsies run the risk of sampling errors as residual tumour cells are often scattered in small clusters, resulting in a sensitivity at 6 weeks post RT of $59.3 \%$ [13].

Radiological assessment faces similar difficulties, and to date there is no consensus regarding the optimal imaging modality in the post treatment setting. The utility of MRI, whilst well established in the initial staging of biopsy-

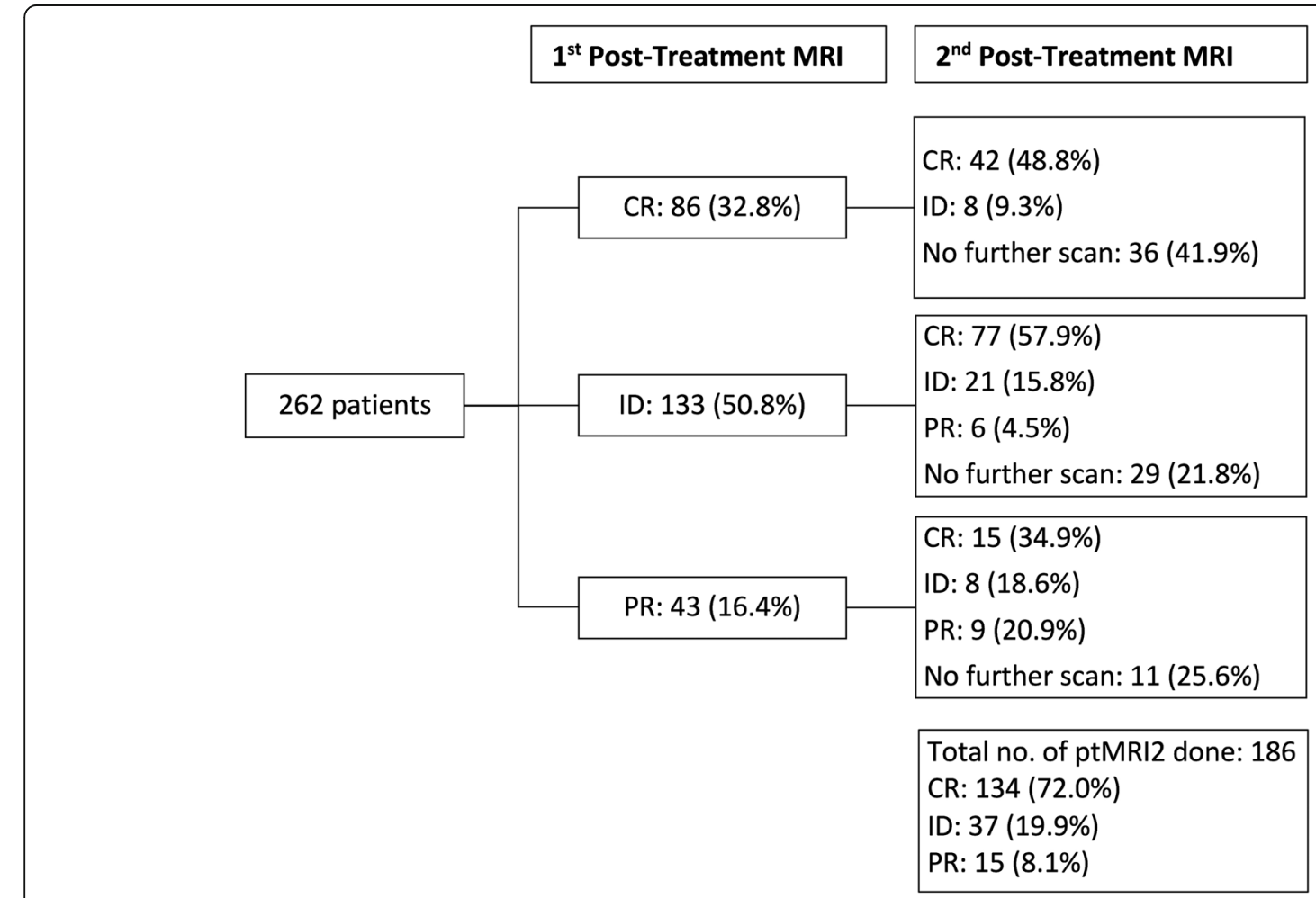

Fig. 1 Post treatment MRI (ptMRI) responses. CR: Complete Response; ID: Indeterminate; PR: Partial Response 
Table 2 Factors associated with complete response (CR) on first post treatment MRI (ptMRI)

\begin{tabular}{|c|c|c|c|c|c|c|}
\hline \multirow[t]{2}{*}{ Variable } & \multicolumn{3}{|c|}{ Univariate analysis } & \multicolumn{3}{|c|}{ Multivariate analysis } \\
\hline & $\mathrm{OR}$ & $95 \% \mathrm{Cl}$ & $P$ & $\mathrm{OR}$ & $95 \% \mathrm{Cl}$ & P \\
\hline Age & 1.00 & $0.98-1.03$ & 0.772 & & & \\
\hline \multicolumn{7}{|l|}{ Gender: } \\
\hline Female & 0.94 & $0.52-1.71$ & 0.84 & & & \\
\hline Male (Ref) & 1 & & & & & \\
\hline \multicolumn{7}{|l|}{ T stage: } \\
\hline $\mathrm{T} 1$ & 3.63 & $1.82-7.20$ & $<0.001$ & 2.96 & $1.07-8.13$ & 0.036 \\
\hline $\mathrm{T} 2$ & 2.83 & $1.07-7.46$ & 0.035 & 2.24 & $0.56-8.90$ & 0.25 \\
\hline T3 & 1.79 & $0.84-3.85$ & 0.134 & & & \\
\hline T4 (Ref) & 1 & & & & & \\
\hline \multicolumn{7}{|l|}{ N stage: } \\
\hline No & 1.87 & $0.76-4.56$ & 0.136 & & & \\
\hline N1 & 0.90 & $0.42-1.94$ & 0.86 & & & \\
\hline N2 & 0.62 & $0.28-1.36$ & 0.24 & & & \\
\hline N3 (Ref) & 1 & & & & & \\
\hline \multicolumn{7}{|l|}{ AJCC Stage: } \\
\hline Stage I & 4.2 & $1.67-10.51$ & 0.002 & 1.78 & $0.39-8.11$ & 0.046 \\
\hline Stage II & 2.31 & $1.12-4.76$ & 0.023 & 0.75 & $0.23-2.46$ & 0.633 \\
\hline Stage III & 1.29 & $0.68-2.44$ & 0.433 & & & \\
\hline Stage IV (Ref) & 1 & & & & & \\
\hline \multicolumn{7}{|l|}{ Treatment modality: } \\
\hline RT alone & 3.12 & $1.45-6.74$ & 0.004 & 1.36 & $0.39-4.72$ & 0.627 \\
\hline ChemoRT & 2.51 & $1.32-4.78$ & 0.005 & 2.46 & $0.98-6.16$ & 0.054 \\
\hline Induction chemo (Ref) & 1 & & & & & \\
\hline
\end{tabular}

OR Odds Ratio, Cl Confidence Interval, AJCC American Joint Committee on Cancer, RT Radiotherapy, Ref Reference

proven NPC, is less clear post treatment. Compared to $\mathrm{CT}$, MRI is able to better differentiate post radiation changes from recurrent tumour and delineate extent of disease $[14,15]$. The identification of skull base erosion is improved with contrast-enhanced fat-suppressed sequences [5].

However, when compared to PET-CT, MRI may be limited in its ability to distinguish between post RT changes often seen in the irradiated nasopharynx and neck e.g. tumour necrosis, tissue fibrosis and inflammation, from true viable tumour. Conversely, changes in tissue metabolism may precede changes in tumour morphology or volume. Liu et al in a systemic review concluded that PET-CT, with its ability for combined functional and anatomic assessment, had superior pooled sensitivity, specificity and overall diagnostic accuracy when compared to both CT and MRI [16]. PET-CT also has the added benefit of uncovering any systemic metastases within the same examination, which can impact on goals of further treatment.
Nevertheless, there are drawbacks to relying solely on PET-CT to uncover residual or recurrent NPC. Disease at the primary site is more accurately demonstrated on MRI rather than PET-CT (92.1\% vs. 85.7\%) according to Comoretto et al. [17]. The latter produced false negative findings especially where there is intracranial extension of disease. False positive results have also been associated with PET-CT when it is done too early post RT due to the residual inflammatory effects causing apparent increased glucose metabolism. It has been suggested that the PET-CT should be done 6 months or later post RT for optimal accuracy (sensitivity and specificity are 92 and $100 \%$ at 6 months or later vs 33 and $64 \%$ within 5 months) [18]. Additionally, cost and resource availability can be limiting factors for the prevailing use of PET-CT. In view of these considerations, it is likely that MRI and PET-CT should be complementary, in order to improve overall diagnostic accuracy for recurrent or residual disease [17]. Our data indicates that, within the boundaries of our institutional practice, most LRR are detected by MRI rather than non-MRI radiological modalities or 
Table 3 Factors associated with indeterminate (ID) status on first post treatment MRI (ptMRI)

\begin{tabular}{|c|c|c|c|}
\hline \multirow[t]{2}{*}{ Variable } & \multicolumn{3}{|c|}{ Univariate analysis } \\
\hline & OR & $95 \% \mathrm{Cl}$ & $P$ \\
\hline Age & 1.00 & $0.99-1.03$ & 0.343 \\
\hline \multicolumn{4}{|l|}{ Gender: } \\
\hline Female & 1.57 & $0.89-2.76$ & 0.119 \\
\hline Male (Ref) & 1 & & \\
\hline \multicolumn{4}{|l|}{ T stage: } \\
\hline $\mathrm{T} 1$ & 0.98 & $0.54-1.77$ & 0.934 \\
\hline $\mathrm{T} 2$ & 0.88 & $0.36-2.15$ & 0.779 \\
\hline T3 & 1.02 & $0.53-1.95$ & 0.964 \\
\hline T4 (Ref) & 1 & & \\
\hline \multicolumn{4}{|l|}{ N stage: } \\
\hline No & 1.38 & $0.57-3.33$ & 0.478 \\
\hline $\mathrm{N} 1$ & 1.71 & $0.82-3.59$ & 0.154 \\
\hline N2 & 1.90 & $0.91-3.97$ & 0.087 \\
\hline N3 (Ref) & 1 & & \\
\hline \multicolumn{4}{|l|}{ AJCC Stage: } \\
\hline Stage 1 & 0.74 & $0.30-1.81$ & 0.509 \\
\hline Stage II & 1.04 & $0.52-2.06$ & 0.919 \\
\hline Stage III & 1.33 & $00.75-20.37$ & 0.329 \\
\hline Stage IV (Ref) & 1 & & \\
\hline \multicolumn{4}{|c|}{ Treatment modality: } \\
\hline RT alone & 0.90 & $0.45-1.79$ & 0.759 \\
\hline \multicolumn{4}{|l|}{ ChemoRT } \\
\hline \multicolumn{4}{|c|}{ Time to first ptMRI (days) } \\
\hline $75-105$ & 2.13 & $1.16-4.12$ & 0.024 \\
\hline 106-135 (Ref) & 1 & & \\
\hline
\end{tabular}

OR Odds Ratio, Cl Confidence Interval, AJCC American Joint Committee on Cancer, RT Radiotherapy, Ref Reference

clinical examination. If salvage surgery or RT is planned for LRR, the superior ability to determine extent of tissue invasion with MRI makes it preferable to PET-CT in guiding resectability or extent of re-treatment required.

Another issue to address is the optimal timing of MRI in view of the potential diagnostic uncertainties post RT. Guidelines suggest varying timing of post treatment imaging ranging from 3 to 12 months [19-21]. The time course of NPC regression after definitive treatment has been studied histologically and radiologically. In 1999 Kwong et al followed 803 NPC patients treated with 3DCRT with or without induction chemotherapy, through 2weekly endoscopic biopsies. 93.2\% achieved histologic remission by 12 weeks post RT. [8] This formed the basis upon which many subsequent studies relied on when scheduling post treatment imaging. Li et al in 2017 challenged this paradigm with data from 556 NPC patients treated definitively with IMRT and followed up with serial MRIs [22]. All MRI scans were reviewed independently by two radiologists with extensive experience in head and neck cancer imaging. In this group, $83.3 \%$ of patients achieved a clinical complete response (cCR) - defined as no evidence of residual tumour or nodal disease based on examination with MRI and flexible nasoendoscopy - at 34 months (early cCR), a figure which increased to $91.4 \%$ at 6-9 months (delayed cCR). Interestingly, prognosis of patients with a delayed $\mathrm{cCR}$ was no different to those with an early $\mathrm{cCR}$, leading the authors to conclude that 6-9 months may be the best time point for assessment of maximal tumour response to IMRT.

The increasing use of IMRT as standard of care, as well as CCRT has been associated with delayed primary tumour regression through mechanisms which to date remain unclear [23]. Whilst results from both Li's and our study both suggest a lag time between histological and radiological tumour regression, a significantly lower proportion of patients in our series (32.8\% vs $83.3 \%)$ achieved a radiological CR on the first ptMRI. This may be explained by the methodology of our study, which looks at the real-world radiology reports, rather than having one or more radiologists retrospectively reviewing imaging and coming to a binary outcome regarding the absence or presence of residual disease. We believe our method reflects real-life clinical practice more closely, where limits of certainty in imaging interpretation is accepted, and collective decision-making is undertaken in a multidisciplinary setting for indeterminate cases. In spite of this uncertainty, having the first ptMRI done at an earlier date may still be worthwhile in order to provide a crucial baseline which can inform future scans. In addition, there may be a window period between 3 and 4 months and 6-9 months where further investigations can lead to earlier diagnosis of residual or recurrent disease, permitting prompt initiation of salvage therapies. Indeed, our study offers evidence that the optimal timing of the first ptMRI should be 4, rather than 3 months to reduce the rates of uncertainty in radiology reports.

We acknowledge that this study has some limitations. Firstly, the retrospective design meant that patients were not followed up based on a standardised protocol. ptMRIs were done at the clinicians' discretion, resulting in a wide date range for the first (32-346 days) and subsequent ptMRIs. Of note, a proportion of patients who had ID/PR status did not go on to have subsequent ptMRIs, reflecting the pattern of real-life clinical practice. Depending on overall clinical suspicion of residual or recurrent disease, these patients may have undergone investigation with other imaging modalities such as PET-CT or had biopsies which confirmed or excluded presence of disease, thereby sidestepping the need for 
further serial MRIs. Secondly, the determination of ptMRI status was based primarily on the authors' review of actual radiology reports rather than centralised review by dedicated radiologist(s). Although the ptMRIs are all reported by radiologists with a special interest in head and neck imaging, their level of experience may be differing.

\section{Conclusion}

MRI has an important role in NPC surveillance compared to other imaging modalities and detected a majority of loco-regional recurrence in our series. However, most patients will require more than one ptMRI to reach a definitive status. The rate of ID scans may be reduced by delaying the first ptMRI to 4 months post-RT.

\begin{abstract}
Abbreviations
3D-CRT: 3-dimensional conformal radiotherapy; AJCC: American Joint Committee on Cancer; CCRT: Concurrent chemoradiation; CR: Complete response; CT: Computed tomography; ID: Indeterminate; IMRT: Intensitymodulated radiotherapy; LRR: Locoregional recurrence; MRI: Magnetic resonance imaging; NPC: Nasopharyngeal carcinoma; PET: Positron emission tomography; PR: Partial response; ptMRI: Post treatment magnetic resonance imaging; RT: Radiotherapy
\end{abstract}

\section{Acknowledgements}

We would like to convey sincere gratitude to our colleagues from the Department of Radiation Oncology at the National University Hospital Cancer Institute who have provided valuable suggestions and support for this study.

\section{Authors' contributions}

TC came up with the study concept and design. He was also involved in data acquisition, manuscript preparation, editing and review. KM collected the data, prepared and edited the manuscript. JT analysed and interpreted the data, and performed statistical analysis. FH edited and reviewed the manuscript. HA helped with data acquisition. All authors read and approved of the final manuscript.

\section{Funding}

Not Applicable.

\section{Availability of data and materials}

The datasets generated and/or analysed during the current study are not publicly available as presently we have not been granted permission by the institutional review board to do so. However, data can be made available from the corresponding author on reasonable request.

\section{Ethics approval and consent to participate}

Ethical approval for this study has been obtained from National Healthcare Group Singapore Domain Specific Review Board (Study Reference No: 2018/ 00630). Waiver of consent has been approved.

\section{Consent for publication}

Not Applicable.

\section{Competing interests}

The authors declare that they have no competing interests.

Received: 21 September 2019 Accepted: 20 February 2020 Published online: 06 March 2020

\section{References}

1. Chua MLK, Wee JTS, Hui EP, Chan ATC. Nasopharyngeal carcinoma. Lancet. 2016;387(10022):1012-24
2. Zhang N, Liang SB, Deng YM, Lu RL, Chen HY, Zhao H, et al. Primary tumor regression speed after radiotherapy and its prognostic significance in nasopharyngeal carcinoma: a retrospective study. BMC Cancer. 2014;14:136. 3. He Y, Zhou Q, Shen L, Zhao Y, Lei M, Wei R, et al. A retrospective study of the prognostic value of MRI-derived residual tumors at the end of intensitymodulated radiotherapy in 358 patients with locally-advanced nasopharyngeal carcinoma. Radiat Oncol. 2015;10:89.

4. Lai V, Khong PL. Updates on MR imaging and (1)(8)F-FDG PET/CT imaging in nasopharyngeal carcinoma. Oral Oncol. 2014;50(6):539-48.

5. Ng SH, Chang TC, Ko SF, Yen PS, Wan YL, Tang LM, et al. Nasopharyngeal carcinoma: MRI and CT assessment. Neuroradiology. 1997;39(10):741-6.

6. King AD, Vlantis AC, Bhatia KS, Zee BC, Woo JK, Tse GM, et al. Primary nasopharyngeal carcinoma: diagnostic accuracy of MR imaging versus that of endoscopy and endoscopic biopsy. Radiology. 2011;258(2):531-7.

7. Chen WS, Li JJ, Hong L, Xing ZB, Wang F, Li CQ. Comparison of MRI, CT and $18 \mathrm{~F}-\mathrm{FDG}$ PET/CT in the diagnosis of local and metastatic of nasopharyngeal carcinomas: an updated meta analysis of clinical studies. Am J Transl Res. 2016;8(11):4532-47.

8. Kwong DL, Nicholls J, Wei WI, Chua DT, Sham JS, Yuen PW, et al. The time course of histologic remission after treatment of patients with nasopharyngeal carcinoma. Cancer. 1999:85(7):1446-53.

9. Lee NY, Zhang Q, Pfister DG, Kim J, Garden AS, Mechalakos J, et al. Addition of bevacizumab to standard chemoradiation for locoregionally advanced nasopharyngeal carcinoma (RTOG 0615): a phase 2 multi-institutional trial. Lancet Oncol. 2012;13(2):172-80.

10. Chua DT, Sham JS, Hung KN, Kwong DL, Kwong PW, Leung LH. Stereotactic radiosurgery as a salvage treatment for locally persistent and recurrent nasopharyngeal carcinoma. Head Neck. 1999;21(7):620-6.

11. Yu KH, Leung SF, Tung SY, Zee B, Chua DT, Sze WM, et al. Survival outcome of patients with nasopharyngeal carcinoma with first local failure: a study by the Hong Kong nasopharyngeal carcinoma study group. Head Neck. 2005; 27(5):397-405.

12. Chao SS, Loh KS, Tan LK. Modalities of surveillance in treated nasopharyngeal cancer. Otolaryngol Head Neck Surg. 2003;129(1):61-4.

13. Kwong DL, Nicholls J, Wei WI, Chua DT, Sham JS, Yuen PW, et al. Correlation of endoscopic and histologic findings before and after treatment for nasopharyngeal carcinoma. Head Neck. 2001;23(1):34-41.

14. Fujii M, Kanzaki J. The role of MRI for the diagnosis of recurrence of nasopharyngeal cancer. Auris Nasus Larynx. 1994;21(1):32-7.

15. Olmi P, Fallai C, Colagrande S, Giannardi G. Staging and follow-up of nasopharyngeal carcinoma: magnetic resonance imaging versus computerized tomography. Int J Radiat Oncol Biol Phys. 1995;32(3):795-800.

16. Liu T, Xu W, Yan WL, Ye M, Bai YR, Huang G. FDG-PET, CT, MRI for diagnosis of local residual or recurrent nasopharyngeal carcinoma, which one is the best? A systematic review. Radiother Oncol. 2007;85(3):327-35.

17. Comoretto M, Balestreri L, Borsatti E, Cimitan M, Franchin G, Lise M. Detection and restaging of residual and/or recurrent nasopharyngeal carcinoma after chemotherapy and radiation therapy: comparison of MR imaging and FDG PET/CT. Radiology. 2008;249(1):203-11.

18. Peng N, Yen S, Liu W, Tsay D, Liu R. Evaluation of the effect of radiation therapy to nasopharyngeal carcinoma by positron emission tomography with 2. Clin Positron Imaging. 2000;3(2):51-6.

19. Chan AT, Gregoire V, Lefebvre JL, Licitra L, Hui EP, Leung SF, et al. Nasopharyngeal cancer: EHNS-ESMO-ESTRO Clinical Practice Guidelines for diagnosis, treatment and follow-up. Ann Oncol. 2012;23(Suppl 7):vii83-5.

20. Colevas AD, Yom SS, Pfister DG, Spencer S, Adelstein D, Adkins D, et al. NCCN guidelines insights: head and neck cancers, version 1.2018. J Natl Compr Cancer Netw. 2018;16(5):479-90.

21. Simo R, Robinson M, Lei M, Sibtain A, Hickey S. Nasopharyngeal carcinoma: United Kingdom national multidisciplinary guidelines. J Laryngol Otol. 2016; 130(S2):S97-\$103.

22. Li WF, Zhang Y, Liu X, Tang LL, Tian L, Guo R, et al. Delayed clinical complete response to intensity-modulated radiotherapy in nasopharyngeal carcinoma. Oral Oncol. 2017;75:120-6.

23. Kong $\mathrm{M}$, Hong SE. Tumor regression patterns based on follow-up duration in patients with head and neck squamous cell carcinoma treated with radiotherapy or chemoradiotherapy. Clin Exp Otorhinolaryngol. 2015;8(4):416-21.

\section{Publisher's Note}

Springer Nature remains neutral with regard to jurisdictional claims in published maps and institutional affiliations. 